\title{
Updated guidelines for the diagnosis and management of aspergillosis
}

\author{
Elizabeth Ann Misch ${ }^{1}$, Nasia Safdar ${ }^{1,2}$ \\ ${ }^{1}$ Department of Medicine, University of Wisconsin-Madison, Madison, Wisconsin, USA; ${ }^{2}$ William S. Middleton Memorial Veterans Hospital, \\ Madison, Wisconsin, USA \\ Correspondence to: Elizabeth Ann Misch, MD. Department of Medicine, University of Wisconsin-Madison, 5th Floor, 1685 Highland Ave., Madison, \\ WI 53705, USA. Email: eamisch@medicine.wisc.edu. \\ Provenance: This is an invited Commentary commissioned by the Section Editor Yan Xu (Department of Respiratory Medicine, Peking Union \\ Medical College Hospital, Peking Union Medical College, Chinese Academy of Medical Sciences, Beijing, China). \\ Comment on: Patterson TF, Thompson GR 3rd, Denning DW, et al. Practice Guidelines for the Diagnosis and Management of Aspergillosis: 2016 \\ Update by the Infectious Diseases Society of America. Clin Infect Dis 2016;63:e1-e60.
}

Submitted Dec 05, 2016. Accepted for publication Dec 09, 2016.

doi: $10.21037 /$ jtd.2016.12.76

View this article at: http://dx.doi.org/10.21037/jtd.2016.12.76

New guidelines for the diagnosis and treatment of infections due to Aspergillus species were published in August of 2016 by the Infectious Diseases Society of America (IDSA) (1), replacing those previously published in 2008 (2). These guidelines incorporate new data published between January 2008 and December 2014. The document provides guidance on non-culture-based diagnosis of Aspergillus infections, discourages the use of combination anti-fungal therapy for primary management, and begins to define the place in therapy for isavuconazole, a new anti-fungal drug of the azole class approved by the U.S. Food and Drug Administration (FDA) in March 2015 to treat aspergillosis and mucormycosis.

The guidelines address 98 clinical questions that cover the three main presentations of Aspergillus infection, invasive, chronic or "saprophytic", and allergic aspergillosis. The executive summary succinctly lists these 98 questions, while the body of the document elaborates the evidence base for each recommendation. The strength of and evidence basis for each recommendation is presented via a Grading of Recommendations, Assessment, Development and Evaluation (GRADE) score (1). Table 1 lists selected key elements of the guideline along with the strength and quality of the evidence in support of each recommendation.

A central recommendation that remains unchanged from the previous iteration of the guidelines is the pre-eminence of voriconazole as first-line therapy for treatment of all invasive forms of aspergillosis. This recommendation has the greatest strength and highest level of evidence when applied to pulmonary aspergillosis, but is less robust when applied to other forms of aspergillosis. Routine use of combination anti-fungal therapy is not recommended for primary therapy [although the use of voriconazole and an echinocandin "can be considered in select patients" (graded as a weak recommendation with moderate-quality evidence)]. Liposomal amphotericin B is retained as an alternative option for primary treatment. For empiric or pre-emptive anti-fungal therapy (disease site unspecified), liposomal amphotericin B, caspofungin, micafungin, or voriconazole are recommended. Anidulafungin is not recommended since no published trials have examined its efficacy as monotherapy for aspergillosis. For prophylaxis of invasive disease in high-risk patients (defined as individuals with graft-versus-host disease or patients with acute myelogenous leukemia or myelodysplastic syndrome and neutropenia), posaconazole is recommended, either in the oral suspension form, as in the previous guideline version, or by extended-release tablet (new recommendation) $(1,2)$. Patients treated for pulmonary aspergillosis in the past should receive secondary prophylaxis during future periods of immune suppression.

Modifications to the previous recommendations for management of aspergillosis include the addition of isavuconazole as an alternative to voriconazole for primary 
Table 1 Key elements of the 2016 U.S. aspergillosis guidelines

\begin{tabular}{|c|c|}
\hline Recommendation & Strength of recommendation, level of evidence* \\
\hline Voriconazole for IPA & Strong, high-quality \\
\hline Voriconazole all other invasive sites of disease & Varies (see full guidelines) \\
\hline Avoid echinocandins & Strong, moderate-quality \\
\hline \multicolumn{2}{|l|}{ Salvage therapy } \\
\hline Amphotericin B lipid complex (ABLC) & Weak, low-quality \\
\hline Caspofungin, micafungin & Weak, moderate-quality \\
\hline Caspofungin, micafungin & Strong, high-quality \\
\hline Voriconazole & Strong, moderate-quality \\
\hline \multicolumn{2}{|l|}{ Prophylaxis in high-risk hosts } \\
\hline Primary: posaconazole & Strong, high-quality \\
\hline $\begin{array}{l}\text { Alternative: voriconazole, itraconazole (suspension), micafungin, or } \\
\text { caspofungin }\end{array}$ & $\begin{array}{l}\text { Voriconazole/itraconazole: strong, moderate-quality; } \\
\text { micafungin/caspofungin: weak, low-quality }\end{array}$ \\
\hline $\begin{array}{l}\text { Lung transplant recipients: inhaled amphotericin B, voriconazole, or } \\
\text { itraconazole }{ }^{\&}\end{array}$ & Strong, moderate-quality ${ }^{\&}$ \\
\hline Resistance testing: not routinely recommended & Strong, moderate-quality \\
\hline \multicolumn{2}{|l|}{ Galactomannan and 1,3- $\beta$-D-glucan } \\
\hline $\begin{array}{l}\text { Use in high-risk patients (HSCT recipients with neutropenia and patients with } \\
\text { hematologic malignancy receiving chemotherapy) }\end{array}$ & $\begin{array}{l}\text { For diagnosis: strong, high-quality; for preemptive } \\
\text { treatment: strong, moderate-quality }\end{array}$ \\
\hline Not recommended in other groups (organ transplant, CGD) & Strong, high-quality \\
\hline $\begin{array}{l}\text { Aspergillus PCR: possibly useful, if available. Non-standardized, not U.S. } \\
\text { FDA-approved, and lacking demonstration of clinical utility in large studies. } \\
\text { May use in individual cases, if combined with other diagnostic and clinical data }\end{array}$ & Careful use acceptable: strong, moderate-quality \\
\hline \multicolumn{2}{|l|}{ Treatment of non-invasive disease } \\
\hline CCPA, no symptoms: observe & Weak, low-quality \\
\hline $\begin{array}{l}\text { CCPA, with symptoms: } 6 \text { months itraconazole or voriconazole (preferred); } \\
\text { posaconazole (third-line) }\end{array}$ & $\begin{array}{l}\text { Itraconazole or voriconazole: strong, high-quality; } \\
\text { posaconazole: strong, moderate-quality }\end{array}$ \\
\hline
\end{tabular}

Table 1 (continued) 
Table 1 (continued)

\begin{tabular}{ll}
\hline Recommendation & Strength of recommendation, level of evidence* \\
\hline Aspergilloma & Strong, moderate-quality \\
If stable: observe & Strong, moderate-quality \\
If hemoptysis: surgically resect & \\
ABPA, oral itraconazole & Weak, low-quality \\
For asthmatics with bronchiectasis or mucus impaction, with symptoms & \\
while on steroids & Weak, low-quality \\
For CF patients with declining lung function or many exacerbations & \\
Allergic fungal rhinosinusitis & Strong, moderate-quality \\
(I) Polypectomy and sinus washout & Strong, moderate-quality \\
(II) Intranasal steroids & Weak, low-quality \\
(III) Oral anti-mold therapy if refractory to (I) or (II) &
\end{tabular}

Adapted from: Patterson TF, Thompson GR 3rd, Denning DW, et al. Practice Guidelines for the Diagnosis and Management of Aspergillosis: 2016 Update by the Infectious Diseases Society of America. Clin Infect Dis 2016;63:e1-e60. *, see p.2 of the full guidelines (1) for explanation of this scoring system; \#, "empiric therapy" is recommended is for "high risk patients with prolonged neutropenia (>10 days) who remain persistently febrile despite broad-spectrum antibiotic therapy"; "preemptive therapy" is recommended for "asymptomatic or febrile, highrisk patients" in whom fungal biomarkers are elevated; \&, voriconazole or itraconazole is recommended over inhaled amphotericin B (weak recommendation; low-quality) for single lung recipients, in patients with mold colonization before or after transplant, or patients with fungal sinusitis. CGD, chronic granulomatous disease; HSCT, hematopoietic stem cell transplant; PCR, polymerase chain reaction; FDA, Food and Drug Administration; CCPA, chronic cavitary pulmonary aspergillosis; ABPA, allergic bronchopulmonary aspergillosis; CF, cystic fibrosis.

treatment of pulmonary aspergillosis (along with liposomal amphotericin B) and the use of micafungin for pre-emptive anti-fungal therapy [see Table 1 and (1)]. Itraconazole has been removed as an acceptable agent for empiric or preemptive therapy. For invasive pulmonary aspergillosis (IPA), amphotericin B lipid complex (ABLC), caspofungin, micafungin, posaconazole and itraconazole have been relegated to salvage therapy. For Aspergillus infections of the eye, previously treated with intravitreal amphotericin $B$ alone, systemic voriconazole is now recommended, in combination with intravitreal amphotericin B or voriconazole and partial vitrectomy. For prophylaxis, caspofungin is newly listed as an alternative agent $(1,2)$. In addition, the guidelines strengthen the argument for therapeutic drug monitoring of the azole class, especially for voriconazole and posaconazole (oral solution) compared to the 2008 version of the document. Trough levels are now recommended for all azole agents active against Aspergillus (strong recommendation; moderate-quality evidence).

There are few revisions to the guidelines for non-invasive forms of aspergillosis, a category that includes aspergilloma, chronic cavitary pulmonary aspergillosis (CCPA), allergic bronchopulmonary aspergillosis (ABPA), and allergic Aspergillus sinusitis. One modification is that treatment for CCPA is now identical to that for IPA. For allergic rhinosinusitis, the recommendation for polypectomy, sinus washout, and intranasal steroids as a combined primary approach is also new.

For the first time, the guidelines advise reducing mold exposure within the hospital or home environment for the most vulnerable patient groups. Populations at highest risk include persons undergoing allogeneic hematopoietic stem cell transplant (HSCT) or receiving induction chemotherapy for acute leukemia. These highly immunecompromised patients should be placed in enclosed areas engineered to reduce mold exposure, where feasible. Appropriate methods for protection include high-efficiency particulate air filtration, laminar airflow, positive pressure rooms, and a standardized number of air exchanges per hour. Alternatively, in facilities without sophisticated engineering barriers, the guidelines advise using private rooms without conduits to construction sites, and avoiding exposure to plants, soil, or cut flowers. High-risk outpatients should avoid gardening, mulching, and proximity to 
construction sites or renovations. Cancer and transplant centers should perform surveillance for aspergillosis, to identify trends in the incidence of invasive fungal infections and allow early detection of new outbreaks.

Enthusiasm for biologic therapies, such as granulocyte infusions, granulocyte colony-stimulating factor (G-CSF) or granulocyte-macrophage colony-stimulating factor (GM$\mathrm{CSF}$ ), to treat invasive aspergillosis remains low (treatment can be considered in patients with refractory neutropenia and aspergillosis), due to unestablished efficacy in human fungal infection. G-CSF and GM-CSF have not been tested in clinical trials of aspergillosis or other infections, although they are effective in shortening the duration of neutropenia. Recently, macrophage colony-stimulating factor (M-CSF) was shown to be protective against otherwise lethal Aspergillus infection in a mouse model of hematopoietic stem/progenitor cell transplant, in contrast to G-CSF, which had no effect on survival (3).

Three other areas of expanded discussion in the new guidelines include (I) non-culture-based diagnosis of aspergillosis through measurement of fungal cell wall components $[1,3-\beta-\mathrm{D}$-glucan (DG) and galactomannan (GM)] or amplification of Aspergillus by polymerase chain reaction (PCR) in host tissues; (II) testing for resistance; and (III) the role of combination anti-fungal therapy.

Methods for the laboratory diagnosis of aspergillosis have evolved considerably beyond culture and now involve detection of fungal cell wall components in human tissues, particularly within blood and bronchoalveolar lavage fluid (BALf), and amplification of fungal DNA by PCR. It is well known but worth restating that yields from fungal culture can be quite low: only $25-52 \%$ of Aspergillus infections are diagnosed based on growth in culture $(4,5)$. Thus, it is not surprising that nearly $45 \%$ of cases reported in one large case series (4) met criteria for "probable", rather than "proven", disease (6). Nonetheless, researchers and clinicians treat these two categories of patients identically. The guidelines intend for the term "invasive aspergillosis" to apply to probable as well as proven cases, but end up placing disproportionate emphasis on culture-based methods of diagnosis: "With 2 important exceptions, proven or probable infection requires the recovery of an organism. The first exception includes the fairly frequent occurrence of histopathological demonstration of hyphae consistent with Aspergillus species in patients with negative culture results. The other exception consists of fulfilling the diagnostic criteria for probable invasive aspergillosis with a surrogate non-culturebased method (i.e., a positive galactomannan assay or $\beta$-glucan assay result and radiologically compatible CT findings) in an immunocompromised host with clinical findings of infection that constitute the definition of probable invasive aspergillosis." (1).

Clinicians in practice treat many patients in the latter category, for whom the diagnosis of aspergillosis has been made in the appropriate clinical (host) context, on the basis of elevated serum or BALf fungal markers in combination with abnormal chest or other imaging. The guidelines recommend restricting the use of either GM or DG as nonculture-based diagnostic tools in patients with hematologic malignancy or HSCT. The lower sensitivity and specificity of these assays outside these high-risk groups, for example, in solid organ transplant recipients, is well supported by the literature (7-11). Consequently, the use of GM or DG as a tool for the diagnosis of aspergillosis in patients with organ transplantation or chronic granulomatous disease (CGD) is discouraged by the guideline authors. The combined or sequential use of these biomarkers, with or without PCR, to diagnosis invasive aspergillosis is not addressed.

The guidelines stop short of recommending Aspergillus PCR of blood or BALf to aid diagnosis or decisions regarding preemptive anti-fungal therapy, given the lack of standardization of PCR targets and protocols and absence of studies showing clinical utility. Nonetheless, clinicians in the United States are likely to include PCR assays in their diagnostic repertoire, since DNA detection can exceed culture in sensitivity, PCR of blood or BAL specimens has a relatively high negative predictive value for invasive aspergillosis (12), and its lower specificity may be offset when combined with GM. In addition, new PCR assays have the ability to detect specific strains of Aspergillus and common azole-resistance mutations in the Cyp51A gene (13). The combination of GM and PCR may be particularly useful for earlier diagnosis of invasive aspergillosis $(14,15)$.

Evidence suggests that there is increasing resistance to azoles among Aspergillus species in centers outside the United States (16-18). Estimates of mortality attributable to azole-resistant aspergillosis range as high as $88 \%$ (19). Despite this trend, the guidelines, somewhat surprisingly, recommend against resistance testing during primary diagnosis and treatment of aspergillosis. This reluctance arises mostly from technical considerations-the lack of clinically established susceptibility breakpoints for moldsrather than a prediction that Aspergillus resistance is unlikely to appear in the U.S. Information on Aspergillus resistance patterns in the U.S. is scant, and this gap in the literature should be addressed. Combination anti-fungal therapy is also discouraged by the guideline authors, due 
to contradictory results from small studies of combination therapy and little concern to date regarding the emergence of resistance. Results from one large study of combination therapy using anidulafungin and voriconazole for pulmonary aspergillosis were inconclusive, but showed benefit in a post-hoc analysis of a subgroup of patients diagnosed by positive galactomannan and radiographic findings (20).

In summary, in the most recent U.S. guidelines for aspergillosis, voriconazole remains first-line treatment for most forms of the disease. Isavuconazole, a promising drug approved in 2015 for treatment of aspergillosis and mucormycosis, is accorded a limited place in therapy as an alternative to voriconazole. Resistance testing and combination anti-fungal therapy are discouraged in initial management, but may be considered for patients failing treatment. The fungal biomarkers GM and DG are useful diagnostic tools in high-risk groups. PCR is a promising new diagnostic tool, but lack of standardization, commercial assays in the U.S., and proof of clinical utility limit its broad dissemination.

\section{Acknowledgements}

This work is supported by a Veteran's Administrationfunded Patient Safety Center of Inquiry (N Safdar).

\section{Footnote}

Conflicts of Interest: The authors have no conflicts of interest to declare.

\section{References}

1. Patterson TF, Thompson GR 3rd, Denning DW, et al. Practice Guidelines for the Diagnosis and Management of Aspergillosis: 2016 Update by the Infectious Diseases Society of America. Clin Infect Dis 2016;63:e1-e60.

2. Walsh TJ, Anaissie EJ, Denning DW, et al. Treatment of aspergillosis: clinical practice guidelines of the Infectious Diseases Society of America. Clin Infect Dis 2008;46:327-60.

3. Kandalla PK, Sarrazin S, Molawi K, et al. M-CSF improves protection against bacterial and fungal infections after hematopoietic stem/progenitor cell transplantation. J Exp Med 2016;213:2269-79.

4. Kontoyiannis DP, Marr KA, Park BJ, et al. Prospective surveillance for invasive fungal infections in hematopoietic stem cell transplant recipients, 2001-
2006: overview of the Transplant-Associated Infection Surveillance Network (TRANSNET) Database. Clin Infect Dis 2010;50:1091-100.

5. Neofytos D, Horn D, Anaissie E, et al. Epidemiology and outcome of invasive fungal infection in adult hematopoietic stem cell transplant recipients: analysis of Multicenter Prospective Antifungal Therapy (PATH) Alliance registry. Clin Infect Dis 2009;48:265-73.

6. De Pauw B, Walsh TJ, Donnelly JP, et al. Revised definitions of invasive fungal disease from the European Organization for Research and Treatment of Cancer/ Invasive Fungal Infections Cooperative Group and the National Institute of Allergy and Infectious Diseases Mycoses Study Group (EORTC/MSG) Consensus Group. Clin Infect Dis 2008;46:1813-21.

7. Husain S, Kwak EJ, Obman A, et al. Prospective assessment of Platelia Aspergillus galactomannan antigen for the diagnosis of invasive aspergillosis in lung transplant recipients. Am J Transplant 2004;4:796-802.

8. Kwak EJ, Husain S, Obman A, et al. Efficacy of galactomannan antigen in the Platelia Aspergillus enzyme immunoassay for diagnosis of invasive aspergillosis in liver transplant recipients. J Clin Microbiol 2004;42:435-8.

9. Fortún J, Martín-Dávila P, Alvarez ME, et al. False-positive results of Aspergillus galactomannan antigenemia in liver transplant recipients. Transplantation 2009;87:256-60.

10. Ku NS, Han SH, Choi JY, et al. Diagnostic value of the serum galactomannan assay for invasive aspergillosis: it is less useful in non-haematological patients. Scand J Infect Dis 2012;44:600-4.

11. Singh N, Winston DJ, Limaye AP, et al. Performance Characteristics of Galactomannan and $\beta$-d-Glucan in High-Risk Liver Transplant Recipients. Transplantation 2015;99:2543-50.

12. Eigl S, Hoenigl M, Spiess B, et al. Galactomannan testing and Aspergillus PCR in same-day bronchoalveolar lavage and blood samples for diagnosis of invasive aspergillosis. Med Mycol 2016. [Epub ahead of print].

13. Chong GM, van der Beek MT, von dem Borne PA, et al. PCR-based detection of Aspergillus fumigatus Cyp 51A mutations on bronchoalveolar lavage: a multicentre validation of the AsperGenius assay® in 201 patients with haematological disease suspected for invasive aspergillosis. J Antimicrob Chemother 2016;71:3528-35.

14. Aguado JM, Vázquez L, Fernández-Ruiz M, et al. Serum galactomannan versus a combination of galactomannan and polymerase chain reaction-based Aspergillus DNA detection for early therapy of invasive aspergillosis in high- 
risk hematological patients: a randomized controlled trial. Clin Infect Dis 2015;60:405-14.

15. Hasseine L, Cassaing S, Robert-Gangneux F, et al. High negative predictive value diagnostic strategies for the reevaluation of early antifungal treatment: A multicenter prospective trial in patients at risk for invasive fungal infections. J Infect 2015;71:258-65.

16. Steinmann J, Hamprecht A, Vehreschild MJ, et al. Emergence of azole-resistant invasive aspergillosis in HSCT recipients in Germany. J Antimicrob Chemother 2015;70:1522-6.

17. Chowdhary A, Sharma C, Hagen F, et al. Exploring azole antifungal drug resistance in Aspergillus fumigatus with special reference to resistance mechanisms. Future Microbiol 2014;9:697-711.

18. Wiederhold NP, Patterson TF. Emergence of Azole Resistance in Aspergillus. Semin Respir Crit Care Med 2015;36:673-80.

19. van der Linden JW, Snelders E, Kampinga GA, et al. Clinical implications of azole resistance in Aspergillus fumigatus, The Netherlands, 2007-2009. Emerg Infect Dis 2011;17:1846-54.

20. Marr KA, Schlamm HT, Herbrecht R, et al. Combination antifungal therapy for invasive aspergillosis: a randomized trial. Ann Intern Med 2015;162:81-9.

Cite this article as: Misch EA, Safdar N. Updated guidelines for the diagnosis and management of aspergillosis. J Thorac Dis 2016;8(12):E1771-E1776. doi: 10.21037/jtd.2016.12.76 